American Journal of Applied Sciences 3 (9): 2011-2019, 2006

ISSN 1546-9239

(c) 2006 Science Publications

\title{
Mesoscopic Damage Based Model for Plane Concrete under Static and Dynamic Loadings
}

\author{
M. Labibzadeh and S. A. Sadrnejad \\ Department of Civil Engineering, K.N. Toosi University of Technology, Tehran, Iran
}

\begin{abstract}
The constitutive equations for the mechanical behavior of concrete capable of seeing damage effects or crack growth procedure under loading/unloading/reloading was developed upon microplane framework. The proposed damage formulation has been built on the basis of five fundamental types of stress/strain combinations, which essentially may occur on any of microplanes. Model verification under different loading/unloading/reloading stress/strain paths has been examined. The proposed model is capable of presenting pre-failure history of stress/strain progress on different predefined sampling planes through material. Many of mechanical behavior aspects happen during plasticity such as induced anisotropy, rotation of principal stress/strain axes, localization of stress/strain and even failure mechanism are predicted upon a simple rational way and can be presented.
\end{abstract}

Key words: Microplane, damage, concrete, model

\section{INTRODUCTION}

A survey through the published scientific papers concern with the object of constitutive modeling of geomaterials, all models are categorized into two main classes: continuous and discontinuous models. In the first class, it is postulated that overall mass of material is continuous and no crack/rupture/gap or fracture are allowed. On the other hand in the last class, material assumed to act as an assemblage of the discrete particles, which can effect on the movements of each other's. The continuous models in turn consist of two large groups: macroscopic models in the context of damage and plasticity theory or combination of both and mesoscopic models such as microplane or multilaminate models. However discontinuous models are placed into one category as microscopic models such as discrete particle models. The macroscopic models concern with the definition of relations between stress and strain tensors (structural scale) and the mesoscopic models deals with the same object but in the different way. The latter capture this goal by assigning of the relations between the stress and strain components of the different planes with prescribed orientations 'called micro or multi planes'. Finally the microscopic models concern with the discrete particle models consisting of convex polygons that is able to withstand a limited cohesion (granular scale). A description of contacts of particles as well as a bond formulation between them could lead to forces induced by particle movements. These forces are inserted into the equations of motion, which are solved numerically based on the discrete element methodology.

Different continuous and discontinuous models have been developed for the numerical simulation of geo and geo-like materials and used upon the objective of the study. Microscopic discontinuous models like particle models are able to represent localization within a material in a straightforward, natural manner. However its application for the daily engineering practices still seems to be ambitious. On the other hand, invariant-based macroscopic continuous models lose some of the important features of material behavior because they are basically not able to capture and store the data properties in the different directions around a material point. In this situation, it seems to be a rational solution to use mesoscopic models, which are placed, in the midway of macro to micro scale study of the material behavior.

Based on the above argument, in this research, we pay our attention for the mesoscopic models (micro or multi plane models), which nowadays are considered as powerful tools for numerical simulation of the geo and geo-like materials.

Slip planes to microplanes: The basic idea, namely that of the constitutive material behavior as a relation between strain and stress tensors can be "assembled" from the behavior of material on the planes with different orientations within the material such as slip planes. Taylor's idea was formulated in detail by Batdorf and Budiansky ${ }^{[1]}$. This theory was soon recognized as the most realistic constitutive model for plastic-hardening metals. It was refined in a number of subsequent works. It was used in arguments about the physical origin of strain hardening and was shown to allow easy modeling of anisotropy as well as the vertex effects for loading increment to the side of a radial path in stress space. All the formulations considered that only the inelastic shear strains ('slips'), with no inelastic 
normal strain, were taking place on what is now called the 'microplanes'. The theory was also adapted to anisotropic rocks and soils under the name "multi laminate model".

It is interesting to note that in all these works there is a common assumption that the planes of plastic slip in the material (in those studies called the 'slip-planes' and here in this article called the 'microplanes') to be constrained statically to the stress ('macro-stress') tensor $\sigma_{i j}$. Bažant and coworkers were interested in this domain of research and tried to simulate the strain softening of geo and geo-like materials with such a theory, which was not of interest in the aforementioned studies. After a short time, they concluded that under the assumption of the static constraint, a strainsoftening constitutive law for the microplane makes the material unstable even if $\varepsilon_{i j}$ is prescribed. They suggested that in replace of static constraint, the kinematic constraint could be used. In the kinematic constraint approach, the strain tensor instead of stress tensor is projected on the planes.

Approach: Equilibrium and compatibility: As it is mentioned in the previous section, before 1984, the mesoscopic models (called slip-planes or multilaminate models) were developed based on the static constraint formulation. In this approach, first the macro-stress tensor is projected on the microplanes. Then by introducing on-plane constitutive laws, the micro-strain vectors are defined. Finally the macrostrain tensor is obtained by using simple superposition of all the micro-strain vectors. So, with some original thinking about this method, it can be easily recognized that nevertheless the equilibrium condition is established with this method in a straightforward manner through the projection of stress tensor on the microplanes, the prove of satisfaction of compatibility condition seems to be not yet as a simple task.

This ambiguity has been removed from 1984 by application of a new method called 'kinematic constraint approach'. In this method, which first introduced by Bažant and his accompanied team, instead of the stress tensor, the strain tensor is projected on the microplanes. So, the compatibility condition is satisfied automatically. Then, after defining the microstress vectors by introducing on-plane constitutive relations between micro-strain and micro-stress vectors, the macro-stress tensor will be calculated from all micro-stress vectors using the principle of virtual work instead of a simple superposition rule. Consequently, in this approach also the equilibrium condition is forcedly established.

A novel microplane damage formulation: After the above argument about the mesoscopic models, in this section we are going to present a new formulation which is a combination of microplane theory with kinematic constraint and damage theory. Then we will attempt to apply it for the simulation of the behavior of plane concrete.

Microplane formulation with kinematic constraint: The orientation of a microplane is characterized by the unit normal $n$ of components $n_{i}$ (indices $i$ and $j$ refer to the components in Cartesian coordinates $\mathrm{x}_{\mathrm{i}}$ ). In the formulation with a kinematic constraint, which makes it possible to describe softening behavior of plane concrete in a stable manner, the strain vector $\vec{\varepsilon}_{N}$ on the microplane (Fig. 1) is the projection of the macroscopic strain tensor $\varepsilon_{i j}$.

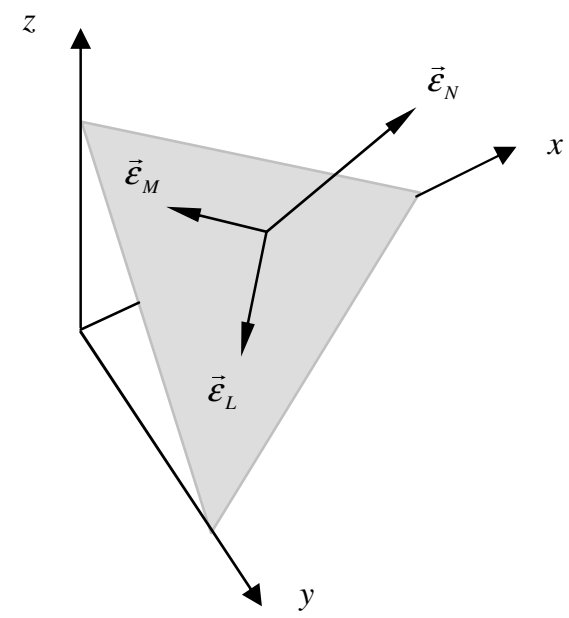

Fig. 1: Strain components on a microplane

So the components of this vector are $\varepsilon_{N i}=\varepsilon_{i j} n_{j}$. The normal strain on the microplane is $\varepsilon_{N}=n_{i} \varepsilon_{N i}$, that is $\varepsilon_{N}=N_{i j} \varepsilon_{i j} ; N_{i j}=n_{i} n_{j}$

Where repeated indices imply summation over $\mathrm{I}=1,2,3$. The mean normal strain, called the volumetric strain $\varepsilon_{V}$ and the deviatoric strain $\varepsilon_{D}$ on the microplane can also be introduced, defined as follows: $\varepsilon_{V}=\varepsilon_{K K} / 3 ; \varepsilon_{D}=\varepsilon_{N}-\varepsilon_{V}$

This separation of $\varepsilon_{V}$ and $\varepsilon_{D}$ is useful when the effect of the hydrostatic pressure for a number of cohesive frictional materials, such as concrete, needs to be captured.

To characterize the shear strains on the microplane (Fig. 1), we need to define two coordinate directions M and L, given by two orthogonal unit coordinate vectors $\mathrm{m}$ and $\mathrm{l}$ of components $\mathrm{m}_{\mathrm{i}}$ and $\mathrm{l}_{\mathrm{i}}$ lying on the microplane. To minimize directional bias of $\mathrm{m}$ and $\mathrm{l}$ among microplanes, one of the unit vectors $\mathrm{m}$ and 1 tangential to the plane is considered to be horizontal (parallel to $\mathrm{x}$ - y plane).

The magnitude of the shear strain components on the microplane in the direction of $\mathrm{m}$ and 1 are as $\varepsilon_{M}=m_{i}\left(\varepsilon_{i j} n_{j}\right)$ and $\varepsilon_{L}=l_{i}\left(\varepsilon_{i j} n_{j}\right)$. Because of the 
symmetry of tensor $\varepsilon_{i j}$, the shear strain components may be written as follows:

$\varepsilon_{M}=M_{i j} \varepsilon_{i j} ; \quad \varepsilon_{L}=L_{i j} \varepsilon_{i j}$

In which the following symmetry tensors were introduced:

$$
\begin{aligned}
& M_{i j}=\left(m_{i} n_{j}+m_{j} n_{i}\right) / 2 ; \\
& L_{i j}=\left(l_{i} n_{j}+l_{j} n_{i}\right) / 2
\end{aligned}
$$

Once the strain components on each microplane are obtained, the stress components are updated through microplane constitutive laws, which can be expressed in algebraic or differential forms.

In the kinematic constraint microplane models, the stress components on the microplanes are equal to the projections of the macroscopic stress tensor $\sigma_{i j}$ only in some particular cases, when the microplane constitutive laws are specifically prescribed in a manner such that this condition can be satisfied. This happens for example in the case of elastic laws at the microplane level, defined with elastic constants chosen so that the overall macroscopic behavior is the usual elastic behavior $^{[2]}$. In general, the stress components determined independently on the various microplanes will not be related to one another in such a manner that they can be considered as projections of a macroscopic stress tensor. Thus the static equivalence or equilibrium between the microlevel stress components and macrolevel stress tensor must be enforced by other means. This can be accomplished ${ }^{[3]}$ by application of the principle of virtual work, yielding

$$
\begin{aligned}
& \sigma_{i j}=\sigma_{V} \delta_{i j}+ \\
& \frac{3}{2 \pi} \int_{\Omega}\left[\sigma_{D}\left(N_{i j}-\frac{\delta_{i j}}{3}\right)+\sigma_{L} L_{i j}+\sigma_{M} M_{i j}\right] d \Omega
\end{aligned}
$$

Where $\Omega$ is the surface of a unit hemisphere, $\sigma_{V}$ and $\sigma_{D}$ are the volumetric and deviatoric part of normal stress component and $\sigma_{L}$ and $\sigma_{M}$ are as shear stress components on the microplanes respectively. Equation (5) is based on the equality of the virtual work inside a unit sphere and on its surface.

The integration in equation (5), is performed numerically by Gaussian integration using a finite number of integration points on the surface of the hemisphere. Such an integration technique corresponds to considering a finite number of microplanes, one for each integration point. An approximate formula consisting of 26 integration points is proposed in this study. In the Table 1, direction cosines and weights of the integration points and in Fig. 2, their positions on the surface of the unit sphere are shown.

Using this numerical integration technique for evaluation of integral statement in equation (5) yields: $\sigma_{i j}=\sigma_{V} \delta_{i j}+\frac{3}{2 \pi} \int_{\Omega} s_{i j} d \Omega \approx \sigma_{V} \delta_{i j}+6 \sum_{\mu=1}^{N_{m}} w_{\mu} s_{i j}(\mu)$

$s_{i j}=\sigma_{D}\left(N_{i j}-\frac{\delta_{i j}}{3}\right)+\sigma_{L} L_{i j}+\sigma_{M} M_{i j}$

(6)

In which $\mathrm{N}_{\mathrm{m}}$ is the number of integration points on hemisphere.

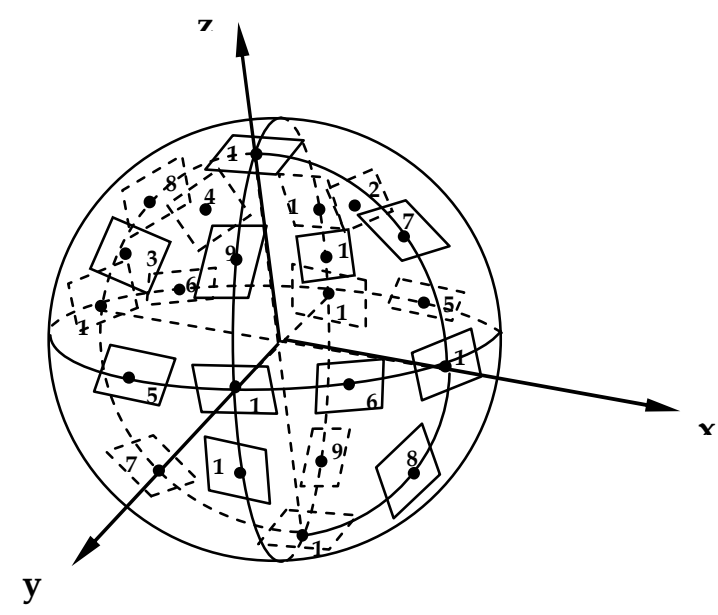

Fig. 2: Position of integration points on the unit sphere' surface

Based on The formulation, macroscopic constitutive matrix in the proposed model is obtained as follows:

$$
\begin{aligned}
& D_{i j k l}=\frac{3}{4 \pi} \int_{\Omega}\left(\frac{E}{1+v}\right) \\
& {\left[\left(N_{i j}-\frac{\delta_{i j}}{3}\right)\left(N_{k l}-\frac{\delta_{k l}}{3}\right)+M_{i j} M_{k l}+L_{i j} L_{k l}\right] d \Omega} \\
& +\frac{E}{1-2 v} \frac{\delta_{k l}}{3} \delta_{i j}
\end{aligned}
$$

In which $E$ and $v$ are as elastic modulus and Poisson's coefficient.

New anisotropy damage function formulation: Total deviatoric part of constitutive matrices is computed from superposition of its counterparts on the microplanes that such counterparts in turn, are calculated based on the damages occurred on each plane depending on its specific loading conditions. This damage is evaluated according to the five separate damage functions; each of them belongs to the particular loading states. This five loading conditions are as follows:

I. hydrostatic compression

II. hydrostatic extension 


\begin{tabular}{|c|c|c|c|c|}
\hline \multicolumn{5}{|c|}{$\begin{array}{l}\text { III. pure shear } \\
\text { IV. shear + compression } \\
\text { V. shear + extension }\end{array}$} \\
\hline \multicolumn{5}{|c|}{ Table 1: Definition of microplanes } \\
\hline $\begin{array}{l}\begin{array}{l}\text { Number } \\
\text { of } \\
\text { integrati } \\
\text { on point }\end{array} \\
\end{array}$ & $\begin{array}{l}\text { Direction } \\
\text { cosine with } \\
\text { respect to x } \\
\text { axis }\end{array}$ & $\begin{array}{l}\text { Direction } \\
\text { cosine with } \\
\text { respect to y } \\
\text { axis }\end{array}$ & $\begin{array}{l}\text { Direction } \\
\text { cosine with } \\
\text { respect to z } \\
\text { axis }\end{array}$ & $\begin{array}{l}\text { Weight of } \\
\text { integratio } \\
\mathrm{n} \text { point }\end{array}$ \\
\hline \multirow[t]{2}{*}{$\frac{011}{1}$} & 1 & 1 & 1 & 27 \\
\hline & $\sqrt{3}$ & $\sqrt{3}$ & $\sqrt{3}$ & 840 \\
\hline \multirow[t]{2}{*}{2} & 1 & 1 & 1 & 27 \\
\hline & $\overline{\sqrt{3}}$ & $\sqrt{3}$ & $\sqrt{3}$ & 840 \\
\hline \multirow[t]{2}{*}{3} & 1 & 1 & 1 & 27 \\
\hline & $-\overline{\sqrt{3}}$ & $\overline{\sqrt{3}}$ & $\overline{\sqrt{3}}$ & 840 \\
\hline \multirow[t]{2}{*}{4} & 1 & 1 & 1 & 27 \\
\hline & $\overline{\sqrt{3}}$ & $\sqrt{3}$ & $\sqrt{3}$ & $\overline{840}$ \\
\hline \multirow[t]{2}{*}{5} & 1 & 1 & 0 & 32 \\
\hline & $\sqrt{2}$ & $\sqrt{2}$ & & $\overline{840}$ \\
\hline \multirow[t]{2}{*}{6} & 1 & 1 & 0 & 32 \\
\hline & $\sqrt{2}$ & $\sqrt{2}$ & & $\overline{840}$ \\
\hline \multirow[t]{2}{*}{7} & 1 & 0 & 1 & 32 \\
\hline & $\sqrt{2}$ & & $\sqrt{2}$ & 840 \\
\hline \multirow[t]{2}{*}{8} & 1 & 0 & 1 & 32 \\
\hline & $\sqrt{2}$ & & $\sqrt{2}$ & $\overline{840}$ \\
\hline \multirow[t]{2}{*}{9} & 0 & 1 & 1 & 32 \\
\hline & & $\sqrt{2}$ & $\sqrt{2}$ & 840 \\
\hline \multirow[t]{2}{*}{10} & 0 & 1 & 1 & 32 \\
\hline & & $\sqrt{2}$ & $\sqrt{2}$ & 840 \\
\hline \multirow[t]{2}{*}{11} & 1 & 0 & 0 & 40 \\
\hline & & & & 840 \\
\hline \multirow[t]{2}{*}{12} & 0 & 1 & 0 & 40 \\
\hline & & & & 840 \\
\hline \multirow[t]{2}{*}{13} & 0 & 0 & 1 & 40 \\
\hline & & & & $\overline{840}$ \\
\hline
\end{tabular}

On each microplane at each time of loading history, there exists one specific loading situation that it may be in one of the five mentioned basic loading conditions. For every five mood, a specific damage function according to the authoritative laboratory test results available in the literature is assigned. Then, for each state of on plane loading, one of the five introduced damage functions will be computed with respect to the history of micro-stress and strain components. These five damage functions are as below:

$\omega_{\mathrm{HC}}=0.0$

$$
\begin{aligned}
& \begin{cases}\omega_{\mathrm{HT}}=0.0 & \text { if } \varepsilon_{\mathrm{eq}} \leq \sqrt{3} \mathrm{a} \\
\omega_{\mathrm{HT}}=1.0-\left(\frac{\sqrt{3} \mathrm{a}}{\varepsilon_{\mathrm{eq}}}\right) \times \exp \left[-\left(\frac{\varepsilon_{\mathrm{eq}}-\sqrt{3} \mathrm{a}}{\mathrm{b}-\sqrt{3} \mathrm{a}}\right)\right] & \text { if } \varepsilon_{\mathrm{eq}}>\sqrt{3} \mathrm{a}\end{cases} \\
& \omega_{\mathrm{SH}}=0.5 \times\left(\omega_{\mathrm{C}}+\omega_{\mathrm{T}}\right) \\
& \omega_{\mathrm{C}}=\mathrm{d} \times \varepsilon_{\mathrm{eq}} \quad \text { if } \varepsilon_{\mathrm{eq}} \leq \mathrm{e} \\
& \left\{\omega_{\mathrm{C}}=\mathrm{f}\left(\varepsilon_{\text {eq }}-\mathrm{e}\right)^{2}+\mathrm{g}\left(\varepsilon_{\text {eq }}-\mathrm{e}\right)+\mathrm{h} \quad \text { if } \mathrm{e}<\varepsilon_{\text {eq }} \leq \mathrm{i}\right. \\
& \omega_{\mathrm{C}}=1.0-\left(\frac{\mathrm{j}}{\varepsilon_{\mathrm{eq}}}\right) \times \exp \left[-\left(\frac{\varepsilon_{\mathrm{eq}}-\mathrm{i}}{\mathrm{k}-\mathrm{i}}\right)\right] \quad \text { if } \varepsilon_{\mathrm{eq}}>\mathrm{i} \\
& \begin{cases}\omega_{\mathrm{T}}=0.0 & \text { if } \varepsilon_{\mathrm{eq}} \leq \mathrm{a} \\
\omega_{\mathrm{T}}=1.0-\left(\frac{\mathrm{a}}{\varepsilon_{\mathrm{eq}}}\right) \times \exp \left[-\left(\frac{\varepsilon_{\mathrm{eq}}-\mathrm{a}}{\mathrm{c}-\mathrm{a}}\right)\right] & \text { if } \varepsilon_{\mathrm{eq}}>\mathrm{a}\end{cases}
\end{aligned}
$$

Parameters a to $\mathrm{k}$ in the above relations are computed according to laboratory results obtained for each specific concrete. In equation (9), $\varepsilon_{\mathrm{eq}}$ is as average strain and in the other relations is as the magnitude of projected deviatoric strain vector on each microplane.

Model parameters: In this formulation we consider just two basic material parameters for ease as elasticity and Poisson's coefficients.

Model algorithm: Figure 3, shows the computational sequence used in the proposed model.

Correlation studies: To establish the validity of the proposed concrete material model, correlation studies of analytical results with experimental evidence from the stress-strain response of concrete specimens under different loading conditions are presented in the following.

Uniaxial compression (UC) test: As can be seen in the Fig. 4, there is a good agreement between the results fulfilled by the proposed model and experimental evidences. The material parameters used in the above analysis are as: $E=25000 \mathrm{MPa}, v=0.20$. In Fig. 5, the volumetric changes of the concrete specimen under uniaxial compressive loading have been compared with the experimental observations experienced by Kupfer and his co-workers in 1969. As it is depicted, there exists an excellent coincidence between analytical and laboratory data. To show more confidence on the capability of the microplanes model during uniaxial compression test, in the Fig. 6, the variation of microstress normal and tangential component values are represented versus the total axial compressive stress.

As can be seen from Fig. 6, during the application of the uniaxial compressive load on the x-axis, the microplane number 11 (Fig. 2) is under just the compressive stress whereas the micro- planes number $9,10,12,13$ which geometrically are located normal to the load direction on the unit sphere are only under the tensile stress. Compressive accompanied with shear 


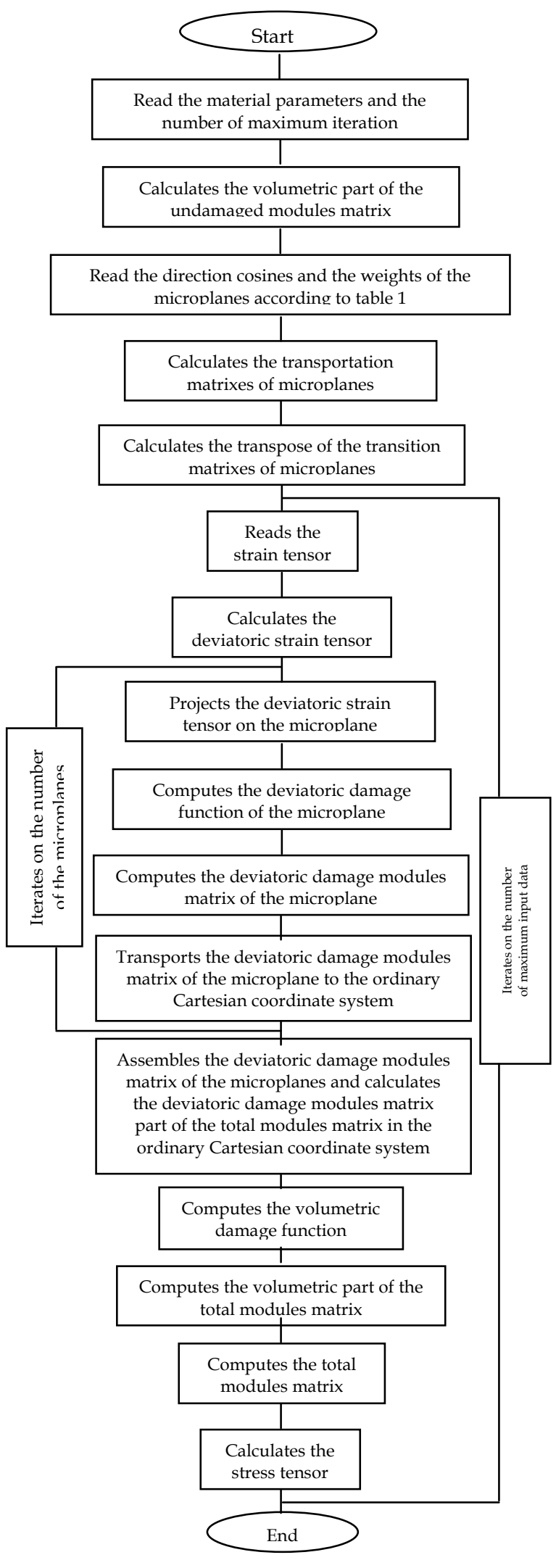

Fig. 3: Operation sequence of the proposed microplane damage model developed in the visual fortran computer language

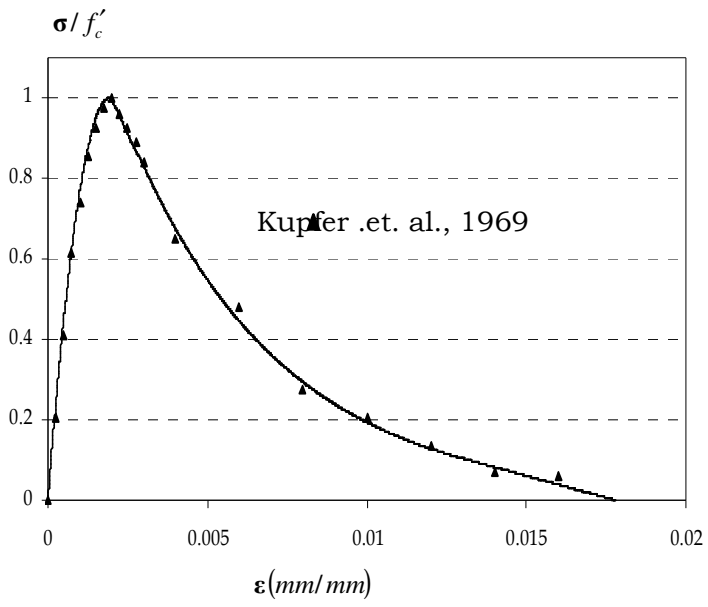

Fig. 4: Uniaxial compression test of concrete obtained with proposed microplane damage model

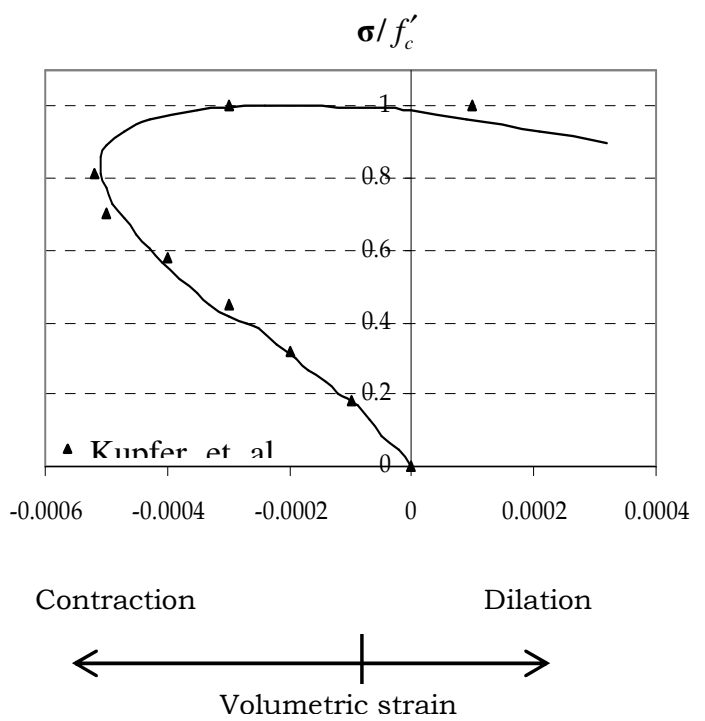

Fig. 5: Volumetric behavior of concrete under uniaxial compressive loading

strains affect the other remaining planes. It is interesting to note that during increase of the uniaxial compressive load, the compressive and shear stress components acting on the microplanes number 1 to 8 increase together with more rise of shear stress at first, but near to the peak stress $\left(f_{c}^{\prime}\right)$ the compressive stress decreases suddenly.

Figure 7 shows the growth of the damage function values of different microplanes during uniaxial compression test of concrete obtained with the proposed model.

As it can be well observed from this figure, damage evolutes faster on the microplanes number $9,10,12,13$ on the unit sphere than the other planes. This is because of the existence of different modes of loading on those planes. In the uniaxial compression test done by the proposed model, the axial compressive load is applied on the $\mathrm{x}$-axes that are normal to the microplane number 11 (Fig. 2). 


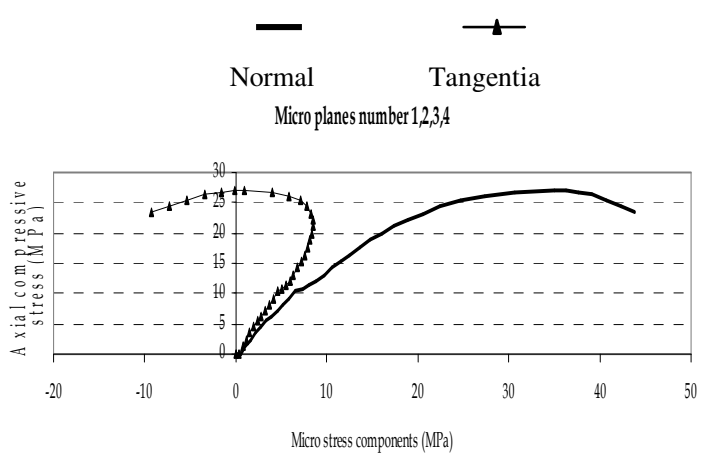

Micro planes number 5,6

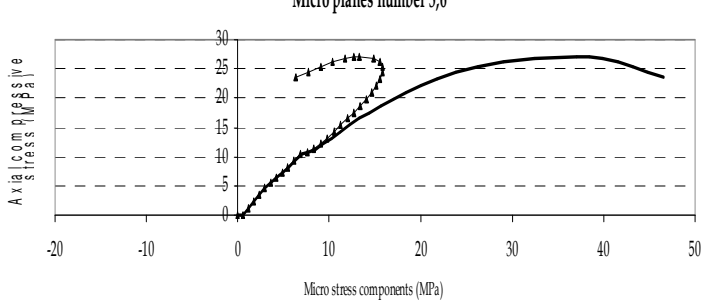

Micro planes number 7,8

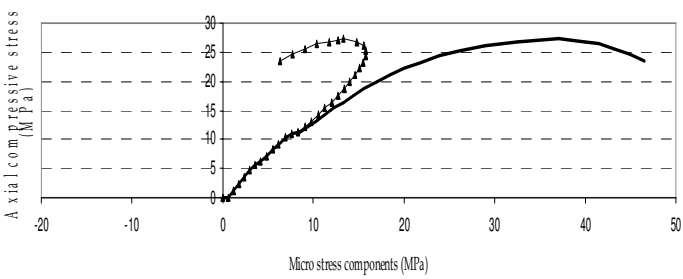

Micro planes number 9,10

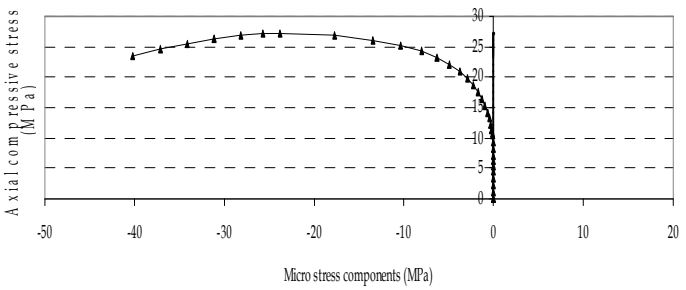

Micro plane number 11

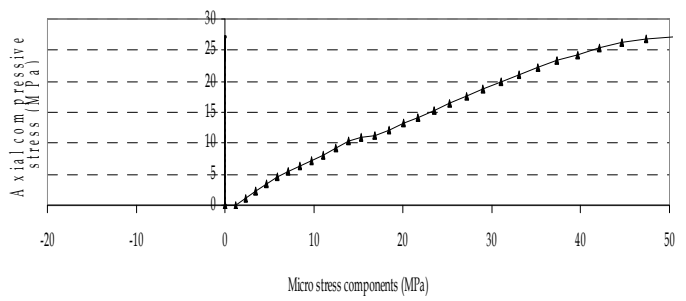

Micro planes number 12,13

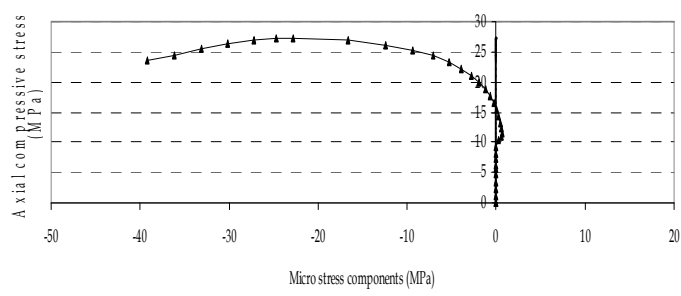

Fig. 6: Variation of micro-stress component values during uniaxial compression test

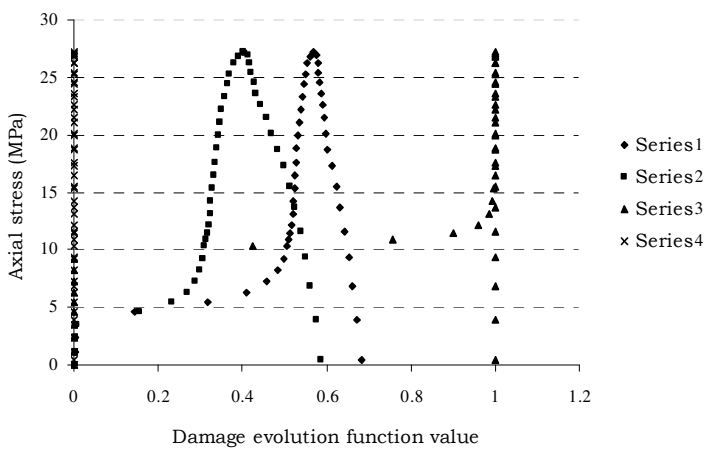

Fig. 7: Comparison of the damage evolution functions on the various microplanes during the axial compressive loading

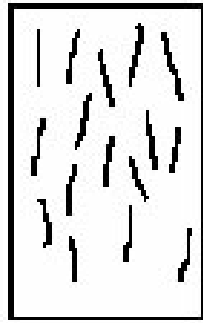

(a)

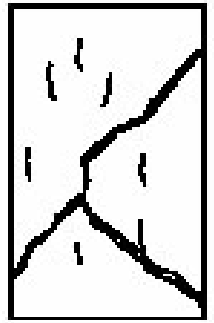

(b)
Fig. 8: Typical failure patterns of cylinders in uniaxial compression test with a) low frictional b) high frictional restraint

So on this plane; there exists only a normal compressive load (mode I) by which no damages could be occurred on it. On the microplanes number 5,6,7,8 there is a shear combined with the normal compressive load (mode IV) causes damages less than the microplanes 1,2,3,4 on which there exists a same mode of loading (mode III). This is because of the fact that on the microplanes number 1,2,3 and 4 the magnitude of the compressive stress component is less than the same component value on the microplanes number 5 to 8 (Fig. 6), so damage growths faster. Finally, on the microplanes number 9,10,12,13 there exist only normal tension loading (mode II), causes the damage growths faster than the all other planes.From the above obtained behaviors of the microplanes, this is introduced from proposed model that in the uniaxial compression test, the damages or cracks can be appeared first on the microplanes number $9,10,12,13$ and then on the microplanes number $1,2,3$ and 4 . This can be so observed in the real situation of the laboratory on the cylindrical concrete specimen. If there is no friction restraint between the surfaces of the loading top/bottom plates and the specimen, the cracks will be appeared differently on the positions of the microplanes number $9,10,12,13$ of the proposed model. Else if the damages on the microplanes number 1, 2, 3 and 4 will be greater and cracks will be initiated first on these planes. This phenomenon is depicted in the Fig. 8. 


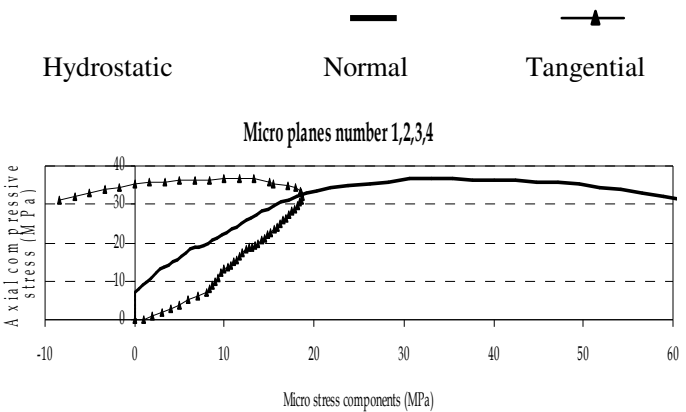

Micro planes number 5,6

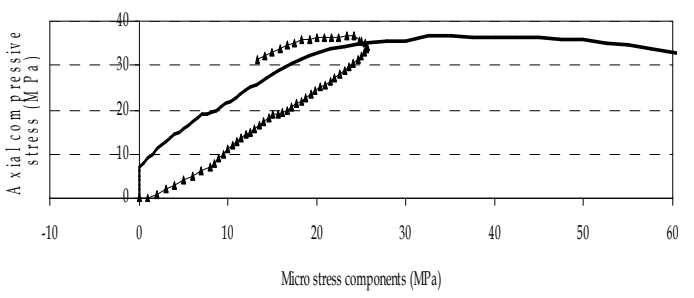

Micro planes number 7,8

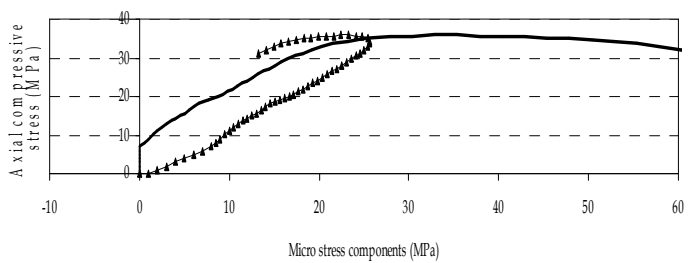

Micro planes number 9,10

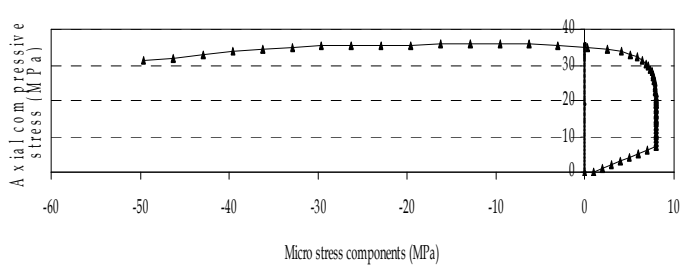

Micro plane number 11

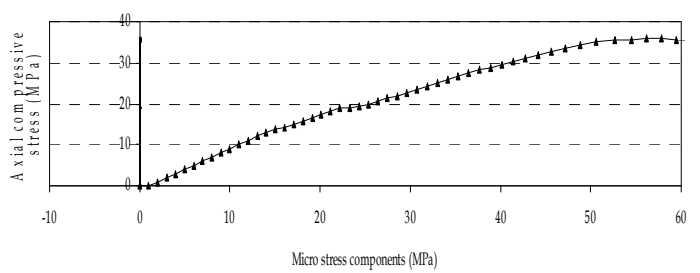

Micro planes number 12,13

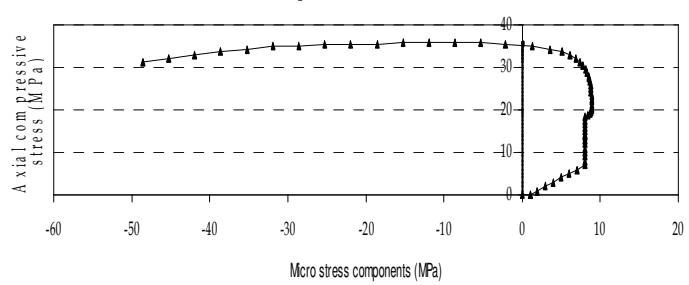

Fig. 9: Variation of micro-stress component values during Conventional Triaxial Compression (CTC) test

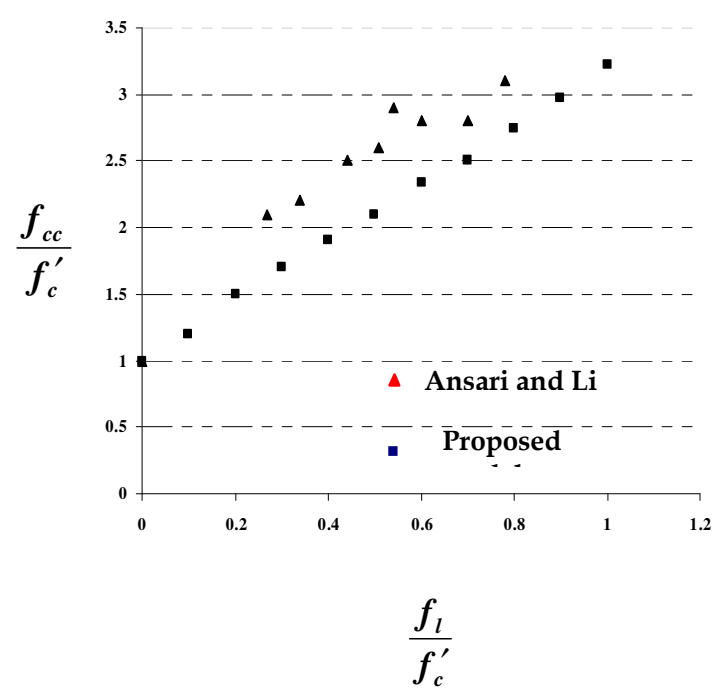

Fig. 10: Different triaxial compression strengths $\boldsymbol{f}_{\boldsymbol{c}}$ obtained with respect to different lateral confining pressures $\boldsymbol{f}_{\boldsymbol{l}}$

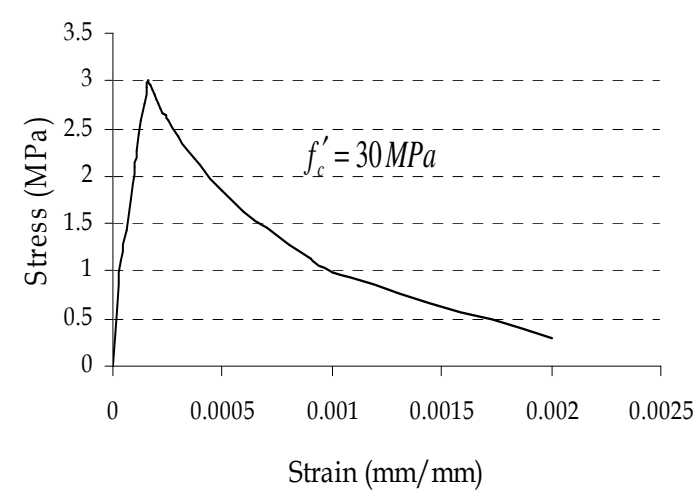

Fig. 11: Behavior of cylindrical concrete specimen under uniaxial tension test obtained with proposed microplane damage model

Convensional triaxial compression (CTC) test: In this test, at first the hydrostatic pressure is applied to the specimen to a certain level and then the axial compression is increased while the lateral or confining pressure held constant. So in this test, up to the certain level of hydrostatic compression there must be no any shear forces on the microplanes. This can be seen in Fig. 9 that shows the evolution of the micro-stress components on the different microplanes during CTC test.

As a result, the effect of lateral confining pressures on the compressive cylindrical strength of concrete specimens simulated by proposed model has been compared with experimental data in Fig. 10.

Uniaxial tension (UT) test: The stress-strain response of the concrete cylindrical specimen under axial tension load is depicted in Fig. 11. 


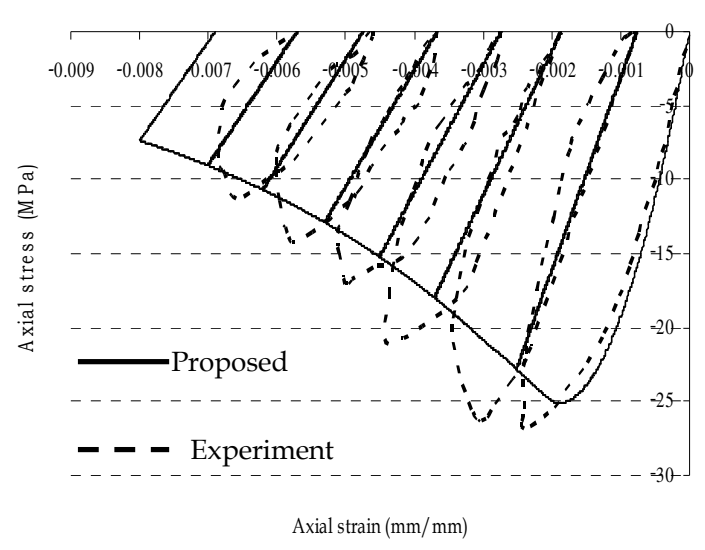

Fig. 12: Cyclic compression test simulation

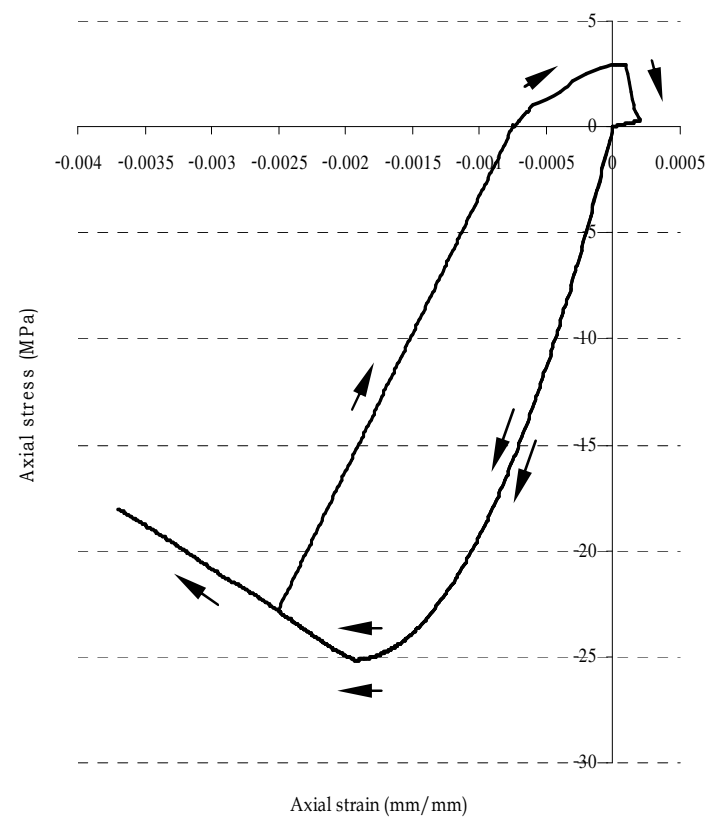

Fig. 13: Complete cyclic test simulation

Cyclic loading: Generally, the most damage models fail to reproduce the irreversible strains and the slopes of the curve in unloading and reloading regions. To overcome this problem, often the plasticity and damage models are combined. In this study, we try to simulate this aspect by the appropriate modifications in the formulation of the proposed microplane damage model. In Fig. 12, the predicted response of the model under cyclic compression test is compared with the experimental results.

There is a good agreement between the analytical and experimental data. Also, in Fig. 13 the behavior of concrete simulated by the proposed model under complete cyclic loading is shown.

\section{CONCLUSION}

A constitutive damage model for the mechanical behavior of concrete under any arbitrary loading conditions was developed using the composition of the theoretical framework of microplane and damage approaches. This model has some characteristics which those distinguishes it from similar challenges have been done recently in this area of researches. Some of those are listed below:

* A new damage formulation has been employed into the microplane model. This damage formulation has been built on the basis of five fundamental force conditions that essentially can be occurred on each microplane. Consequently, any arbitrary change of six strain/stress components on cubic element led to combination of five introduced on plane conditions. Therefore, the proposed model is capable of predicting the concrete behavior under any arbitrary strain/stress path. These five force conditions are as follows:

I. hydrostatic compression

II. hydrostatic extension

III. pure shear

IV. shear + compression

V. shear + extension

The five damage evolution functions which all of them are as functions of equivalent strain were formulated for any of the five force conditions. The equivalent strain for the two first conditions is defined as volumetric strain and for the others it may be considered as magnitude of projected vectors of deviatoric strain tensor on the corresponding microplanes. These damage evolution functions are constructed with respect to the experimental evidences on the concrete specimens under compressive and tensile loading conditions reported by the researchers on the addressed literatures.

In our formulation, according to the damage theory, the value of total damage function in each microplane varies between zero to one, which the first value is related to the undamaged state (no crack initiation on the microplane) and the second value is referred to the totally damaged state (crack opening on the microplane is greater than the specified critical value). So, with respect to this proposed simulation, in each step of numerical calculation, we have one specific damage function value on each microplane according to the crack state on it and consequently we can evaluate the stiffness degradation or rehabilitation at any physical point of material precisely due to consideration of crack state data all around it. Furthermore, because of allocation of three microplane strain components on each plane in this research, one normal and two others tangent to the plane, this is possible to determine the direction of crack growths around a point exactly.

* As soon as any crack initiates and starting to growth on every of 26 microplanes, the stiffness of the plane orthogonal to the crack line gradually decreased. When the crack opening reaches to its critical value, the components of stiffness matrix 
related to the direction of normal to the crack are reduced to zero. If the on-plane loading conditions changes in such a way that crack starts to be closed, when the crack opening reaches to values smaller than critical crack opening, those mentioned stiffness components increase again and go back to its initial values.

* $\quad$ By testing proposed model under various loading conditions, it was observed that for a particular microplane, the shear and extension strain component combination $(\operatorname{mood} V$ ) could result in damages much more than the other situations. After that hydrostatic extension (II), pure shear (III), shear and compression (IV) and hydrostatic compression $(I)$, respectively has smaller effect on damage evolution.

* This novel microplane damage model can simulate behavior of concrete specimen under compressive loadings as well as tensile loadings with a few model parameters requirements.

* Although the proposed model has excellent features such as pre-failure configuration of inside material, final failure mechanism, capability of seeing induced/inherent anisotropy and also any fabric effects on material behavior, the basis of its formulation is simple, logical and has some physical insights that make it convenient to perceive.

\section{REFERENCES}

1. Batdorf, S.B. and B. Budiansky, 1949. A mathematical theory of plasticity based on the concept of slip. Technical Note 1871, National Advisory Committee for Aeronautics.

2. Carol, I. and Z. Bazant, 1997. Damage and plasticity in micro plane theory. Int. J. Solids \& Structures,. 34: 3807-3835.

3. Bazant, Z.P. and P.G. Gambarova, 1984. Crack shear in concrete: Crack band micro plane model. J. Struct. Eng., ASCE, 110: 2015-2036. 